\title{
Activated microglia contribute to neuronal apoptosis in Toxoplasmic encephalitis
}

\author{
Yi-hua Zhang ${ }^{1 \dagger}$, He Chen ${ }^{1,2+}$, Ying Chen ${ }^{2}$, Lu Wang ${ }^{1}$, Yi-hong Cai ${ }^{1}$, Min Li ${ }^{1}$, Hui-qin Wen ${ }^{1,3}$, Jian Du ${ }^{4}$, Ran $\mathrm{An}^{4}$, \\ Qing-li Luo ${ }^{1}$, Xue-long Wang ${ }^{1}$, Zhao-Rong Lun ${ }^{5}$, Yuan-hong $\mathrm{Xu}^{2^{*}}$ and Ji-long Shen ${ }^{1^{*}}$
}

\begin{abstract}
Background: A plethora of evidence shows that activated microglia play a critical role in the pathogenesis of the central nervous system (CNS). Toxoplasmic encephalitis (TE) frequently occurs in HIV/AIDS patients. However, knowledge remains limited on the contributions of activated microglia to the pathogenesis of TE.

Methods: A murine model of reactivated encephalitis was generated in a latent infection with Toxoplasma gondii induced by cyclophosphamide. The neuronal apoptosis in the CNS and the profile of pro-inflammatory cytokines were assayed in both in vitro and in vivo experiments.

Results: Microglial cells were found to be activated in the cortex and hippocampus in the brain tissues of mice. The in vivo expression of interleukin-6 (IL-6), interleukin-1 $\beta$ (IL-1ß), tumor necrosis factor-a (TNF-a), and inducible nitric oxide synthase (iNOS) were up-regulated in TE mice, and accordingly, the neuronal apoptosis was significantly increased. The results were positively correlated with those of the in vitro experiments. Additionally,apoptosis of the mouse neuroblastoma type Neuro2a (N2a) remarkably increased when the N2a was co-cultured in transwell with microglial cells and Toxoplasma tachyzoites. Both in vivo and in vitro experiments showed that minocycline (a microglia inhibitor) treatment notably reduced microglial activation and neuronal apoptosis.
\end{abstract}

Conclusions: Activated microglia contribute to neuronal apoptosis in TE and inhibition of microglia activation might represent a novel therapeutic strategy of TE.

Keywords: Toxoplasmic encephalitis, Microglia, Neuronal apoptosis, Minocycline

\section{Background}

Toxoplasma gondii is an obligate intracellular parasite which infects nearly $30 \%$ of the human population worldwide [1,2]. Infection of immunocompetent individuals with $T$. gondii does not result in dominant clinical disease. In immunocompromised individuals, however, such as HIV/AIDS or those with continuous immunosuppressive medication, may have clinical manifestations or even lifethreatening Toxoplasmic encephalitis (TE) due to reactivation of the chronic quiescent infection [3,4]. For those patients, dormant encysted bradyzoites change into fastreplicating tachyzoites and cause severe disease and even

\footnotetext{
*Correspondence: xyhong1964@163.com; shenjilong53@126.com ${ }^{\dagger}$ Equal contributors

${ }^{2}$ Clinical Laboratory, the First Affiliated Hospital of Anhui Medical University, Hefei, Anhui, China

${ }^{1}$ The Key Laboratory of Zoonoses and Pathogen Biology Anhui, and Department of Parasitology, Anhui Medical University, Hefei, China Full list of author information is available at the end of the article
}

death by damage to the brain [5]. TE is the most vital outcome of toxoplasmosis in immunocompromised patients who may have fever, headache, altered mental state, seizures, weakness, cranial nerve disturbances, sensory abnormalities, cerebellar signs, meningismus, movement disorders, and neuropsychiatric disorders as well $[2,6]$. Previous studies have demonstrated that inflammation potentially is one of the principal contributors to neuronal damage and death in chronic T. gondii infections $[4,7,8]$. So far the underlying mechanism of microglia-involved pathogenesis in TE has not been well elucidated. Microglia are the resident immune cells in the CNS that would be activated in response to injury, inflammation or the presence of pathogens $[9,10]$. Microglia can secrete antiinflammatory cytokines and neurotrophic factors that are potentially neuroprotective [11]. Activated microglia, however, could significantly induce production of a large array of inflammatory cytokines, like interleukin-1 $\beta$ (IL-1 $\beta$ ), 
tumor necrosis factor alpha (TNF- $\alpha$ ) [12] and inducible nitric oxide synthase (iNOS) [13], leading to the neuronal cell damage of inflammatory surroundings. The bystander effect of activated macroglia plays a decisive role in the pathogenesis of traumatic brain injury (TBI), intracerebral hemorrhage ( $\mathrm{ICH})$, manganese poisoning, and Lyme neuroborreliosis (LNB) [14-17].

Without the involvement of lipopolysaccharide (LPS), T. gondii glycosylphosp- hatidylinositols (GPIs), which are known to activate TLR2 and TLR4 receptors, may trigger production of inflammatory cytokines by mouse macrophages [18]. Additionally, Toxoplasma secretes effector molecules of rhoptries(ROPs) and dense granules (GRAs) that have been shown to stimulate inflammatory mediators in macrophage $[12,19]$.

Accumulated evidence shows that $T$. gondii may not directly cause the apoptosis of host cells [20,21], but indirectly renders the cells vulnerable to immune-mediated responses [21]. Infection of T.gondii results in increased levels of inflammatory mediators [22] and microglial nodules [23] in the CNS. Multitudinous studies have focused on the characteristics of systemic immunity in control of $\mathrm{TE}$, and little is known about the role of microglia in the immunopathogenesis of reactivated TE. Herein we unfolded the functions of microglia and revealed the effect of activated microglia in neuronal apoptosis of TE.

\section{Methods}

\section{Parasites and cell lines}

T.gondii Wh6 strain with genotype Chinese 1 (ToxoDB ${ }^{\#}$ ) was isolated as previously described [24]. Cysts were maintained in the brain of chronically infected mice for in vivo infection, whereas tachyzoites were grown in human foreskin fibroblast (HFF) monolayers for in vitro experiments. The BV-2 mouse microglial cell line and mouse neuroblastoma Neuro2a (N2a) were kindly provided by Dr. SH Huang (Department of Immunology, Anhui Medical University) and cultured in Dulbecco's modified eagle's medium (DMEM) supplemented with $10 \%$ fetal bovine serum (FBS, GIBCO), 100U/ml penicillin, $100 \mu \mathrm{g} / \mathrm{ml}$ streptomycin, and $2 \mathrm{mM}$ L-glutamine.

BV-2 cells were seeded at $1 \times 10^{6}$ cells/well in a 12 well plate. After $2 \mathrm{~h}$, the medium was replaced by $1 \%$ FBS and co-cultured with fresh $T$. gondii tachyzoites at an MOI of $1: 1$ for $24 \mathrm{~h}$.

\section{Animals and infection}

Animal use in all experimental procedures was approved by the Institutional Animal Care and Use Committee of Anhui Medical University. BALB/c mice (4-6 weeks old) were randomly assigned into three groups: the control group (Control), the group of Toxoplasmic encephalitis (TE), and the group of TE with minocycline treatment $(\mathrm{TE}+\mathrm{M})$. The mouse model of TE was established as previously described with slight modifications $[25,26]$. Briefly, the cysts from Wh6 strain were prepared by homogenization of the brain tissues in phosphate-buffered saline (PBS). BALB/c mice were intragastrically administered with 30 cysts. After 45 days, mice with latent infection were intraperitoneally given with cyclophosphamide (50 mg/kg, Baxter Oncology GmbH, Germany) to induce recurrence of toxoplasmosis. Simultaneously, mice were administered with minocycline (45 mg/kg, Sigma) and cyclophosphamide $(50 \mathrm{mg} / \mathrm{kg})$ intraperitoneally 45 days post-infection for inhibition of microglia activation. Fifteen days later, all mice of the three groups were euthanized for collection of the brain parenchyma and for subsequent experiments.

\section{Enzyme-linked immunosorbent assay (ELISA)}

IL-1 $\beta$, IL-6, and TNF- $\alpha$ were measured with a commercial kit according to the manufacturer's instructions (4A Biotech Co, Beijing). The supernatant was collected from brain tissue homogenization and BV-2 was co-cultured with T.gondii tachyzoites. Brain tissues (100 mg) from each mouse were homogenized and centrifuged at 12 $000 \mathrm{~g}$ for $15 \mathrm{~min}$. Supernatant was harvested from BV-2 cells co-cultured with tachyzoites (MOI 1:1) for $24 \mathrm{~h}$.

\section{Immunoblotting}

Proteins were extracted from brain tissues $(100 \mathrm{mg})$, BV-2 cells $\left(1 \times 10^{5}\right)$, and N2a cells $\left(1 \times 10^{5}\right)$, respectively, and were subjected to SDS-PAGE. Separated proteins were transferred onto nitrocellulose membranes (Millipore Corp, Billerica, MA) and were blocked with 5\% skimmed milk. After blocking, the membranes were incubated with the primary antibodies, including mouse monoclonal anti-NeuN (1:1 000, Chemicon, Temecula, CA), mouse monoclonal anti-iNOS (1:500, BD, Bio-science), and mouse monoclonal anti-actin (1:500, Santa Cruz, CA). After washing, the membranes were incubated with horseradish peroxidase (HRP)-conjugated secondary antibodies (1:10 000, Zhongshan BioTech Co, China). The protein bands were visualized by using an ECL kit (Super Signal West Pico, Thermo Scientific, USA).

\section{qRT-PCR for cytokines detection}

Total RNAs from brain tissues and BV-2 cells were extracted by using TRIzol reagent (Invitrogen, USA, CA) and reverse-transcribed with cDNA reverse transcription kit (Invitrogen, USA, CA). Semiquantitative PCR was run for cytokine (TNF- $\alpha$, IL-6, and IL-1 $\beta$ ) detection. Primer design and PCR amplification were performed as previously described [27].

\section{Parasite burden}

The mice were euthanised and the brain tissues were harvested. To quantify the parasite burden in the brain 
of each mouse, a $50 \mathrm{mg}$ brain tissue sample taken from each mouse in the respective groups was digested and DNA templates were extracted by using a DNA extraction kit (QIAGEN GmbH, Hilden, Germany) following the instructions of the manufacturer. The concentration or yield of DNA was determined with UV spectrophotometry based on an OD ratio at 260 and $280 \mathrm{~nm}$ (an absorbance ratio of 1.8-2.0). Subsequently, an ABI prism 7500 sequence detection system (Applied Biosystems, Foster City, CA, USA) was used for 40 cycles of polymerase chain reaction (PCR). The cycle threshold values $(\mathrm{Ct})$ indicative of the quantity of the target gene expressed were determined. In this assay, an increase of fluorescent signal above a preset threshold within 38 PCR cycles was considered positive (i.e., $\mathrm{Ct}<38$ ). The thermal cycling conditions were programmed according to the manufacturer's guidance.

\section{Histopathology}

Brain tissues were harvested from mice with chronic T.gondii infection and TE and fixed in 10\% formalin, embedded in paraffin and cut into $4 \mu \mathrm{m}$-thick sections. The sections were stained with hematoxylin and eosin. Histopathology and immunohistochemical studies were carried out to assess TE models based on the diagnostic criteria of TE released by the US Centers for Disease Control and Prevention (CDC) [28].

\section{Immunohistochemical staining}

Immunohistochemical staining was performed as previously described [29] for detection of parasites and microglia. Brain sections were stained with mouse monoclonal anti-Toxoplasma SAG1(1:100, Abcam, Cambridge, UK) and rabbit polyclonal anti-Iba-1(1:1000, Wako, Osaka, Japan) at $4^{\circ} \mathrm{C}$ for overnight. After rinsing with PBS for $3 \times$ $5 \mathrm{~min}$, the sections were incubated with goat anti-mouse IgG and goat anti-rabbit (Zhongshan BioTech Co, China) at 1:75 dilution for $1 \mathrm{~h}$ at room temperature, and then with peroxidase-antiperoxidase-coupled secondary antibodies for $30 \mathrm{~min}$. Finally, the sections were developed with a mixture of 3,3'-diaminobenzine(DAB) and counterstained with hematoxylin. The iNOS was detected using multiple fluorescence immunohistochemistry in vivo and in vitro. Frozen sections of mouse brain tissues were prepared as previously described [30]. The sections were co-stained with the primary antibodies, including mouse monoclonal anti-iNOS (1:500, BD Bioscience, NJ), rabbit polyclonal anti-Iba-1(1:1000, Wako, Osaka, Japan), FITC-mouse monoclonal anti-Toxoplasma SAG1(1:100, ViroStat, Portland, ME, USA), rabbit polyclonal anticaspase-3(1:500, Santa Cruz, CA), at $4^{\circ} \mathrm{C}$ for overnight. After rinsing with PBS for 3-5 min, the matching secondary antibodies (anti-mouse -FITC or anti-rabbitRhodamine, 1:100, Zhongshan BioTech Co, China) were added to the slides and left for $1 \mathrm{~h}$ at room temperature. After rinsing with PBS, the sections were incubated with

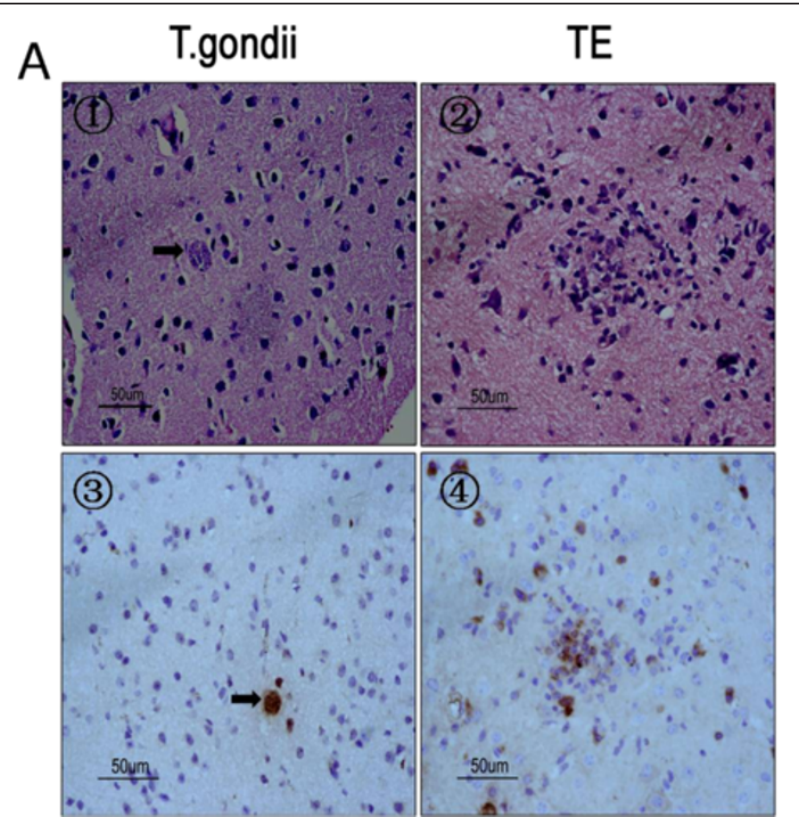

\section{B}

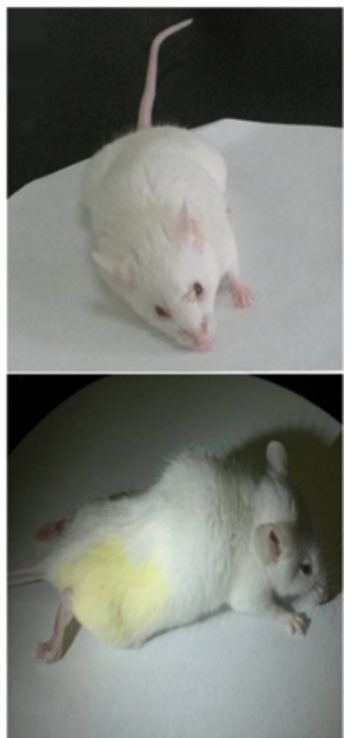

Figure 1 Toxoplasma encephalitis model was established. Microscopic observation of the brain tissues of mice latently infected with cyst-forming T. gondii Wh6 strain (genotype Chinese 1) reactivated by cyclophosphamide administration. (A(1) and (A(3), a single cyst (arrows) in the cortex of chronically infected mice; (A(2) necrosis and inflammatory infiltration noted in foci of recrudescence; (A(4)) free tachyzoites in TE ( $\times 400$ ). The sections were incubated with mouse anti-Toxoplasma SAG1 monoclonal antibody and peroxidase-antiperoxidase-coupled secondary antibody, followed by DAB colorization. Figure $\mathbf{B}$, hemiplegia and paraplegia in model mice with Toxoplasma encephalitis. 
Hoechst for $10 \mathrm{~min}$, and then were observed under fluorescence microscope. To quantify the number of microglia cells labeled with Iba-1, six sections per mouse (from cortex and hippocampus) were examined with microscopy. The images were analyzed with Image Pro Plus 6.0 software.

\section{TUNEL assay}

For TUNEL assay, N2a cells were used for an in vitro experiment. Transwell culture was used to physically separate mouse N2a cells from BV-2 cells. The N2a cells were plated directly into 12 -well tissue culture plates at a density of $5 \times 10^{5}$ parasites and BV-2 cells were plated on transwell filters $(0.4 \mu \mathrm{m}$ pore size; Corning) and then incubated for $24 \mathrm{~h}$ at $37^{\circ} \mathrm{C}$ and $5 \% \mathrm{CO}_{2}$ in DMEM with $1 \%$ FBS. To assess neuroprotective effects, Wh6 tachyzoites and BV-2 cells were plated on transwell filters and treated with minocycline (Sigma, $45 \mu \mathrm{M}$ ). The cells were fixed with $4 \%$ paraformaldehyde for $30 \mathrm{~min}$ and permeabilized with PBS containing 0.4\% Triton X-100 for 15 min. After blocking with 4\% BSA, the cells were then incubated in TUNEL kit (Roche Diagnostics GmbH, Mannheim, Germany) according to the manufacturer's instructions. To assess apoptosis, the numbers of TUNEL stained nuclei were counted by two investigators who worked blindly with regard to the treatments in five randomly selected microscopic fields at $\times 400$ magnification per section.

\section{Statistical analysis}

SPSS (the Statistical Package for Social Sciences) 13.0 (SPSS Inc, Chicago, IL) was used for data analysis. All data are expressed as mean \pm SEM. Differences between groups were assessed by one-way ANOVA and the SNK multiple comparison post-test or Student's $t$ test. Differences were considered statistically significant when $\mathrm{p}<0.05$.

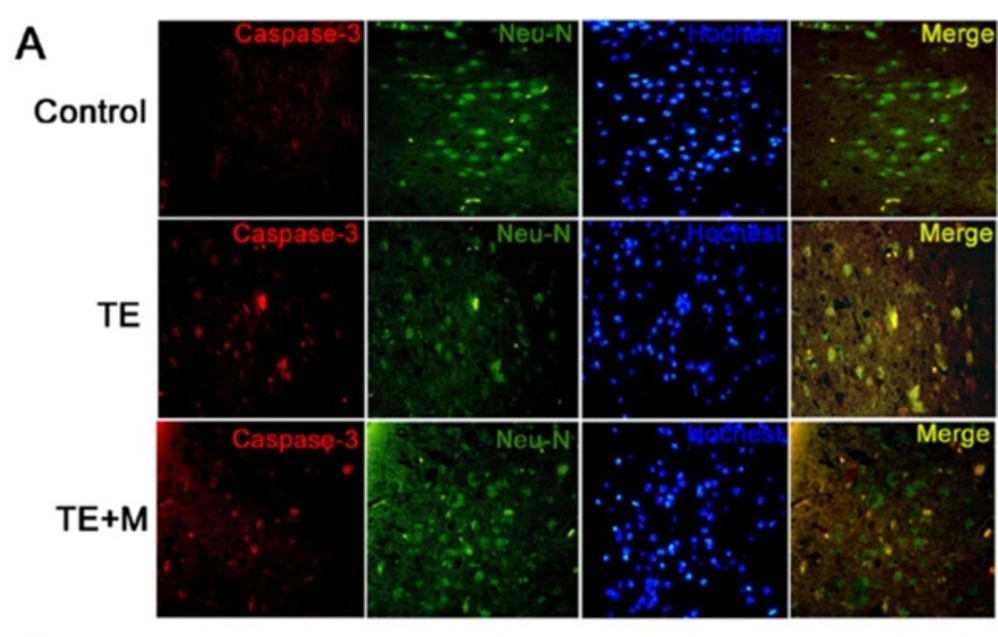

B
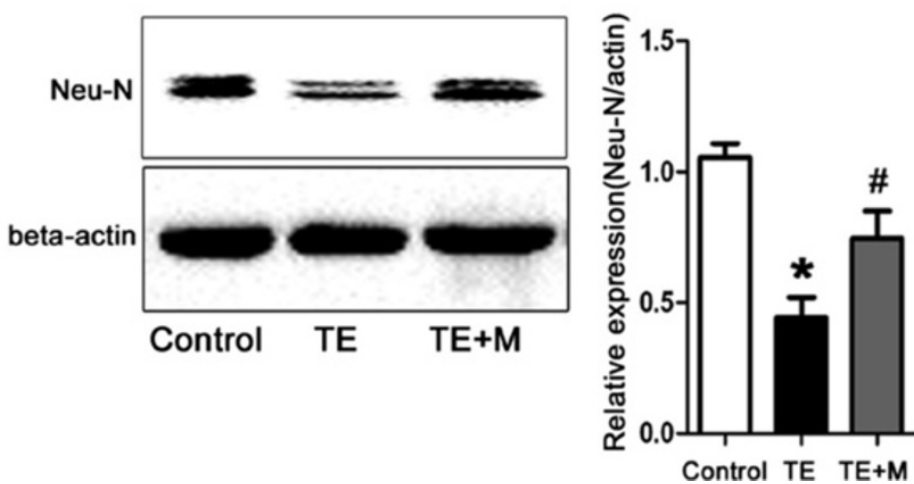

Figure 2 Neuronal apoptosis in the CNS of control, TE, and TE + M mice. (A) Double immunofluorescent staining of caspase-3 (a marker of cell death) and NeuN (a specific marker of neuron cell) demonstrated apoptosis of neuron cells. Brain sections from cortex of control, TE and $\mathrm{TE}+\mathrm{M}$ animal groups were stained with polyclonal caspase-3(red) antibody and monoclonal NeuN antibody (green). Cells were counter-stained with Hoechst to show nuclei (blue). Double immunostaining showed caspase-3 expression in neurons. Increased number of caspase-3 positive cells in TE was reduced significantly in TE + M. (B) Western blotting assay was used for analysis of protein NeuN expression in control and TE and $T E+M$ mice. It showed that the downregulated expression of NeuN in TE was increased significantly after treatment with minocyline (TE + M). Values represent the means \pm SEM from six animals in each group (*, $p<0.01$ vs. Control. \#, $p<0.01$ vs. TE). 


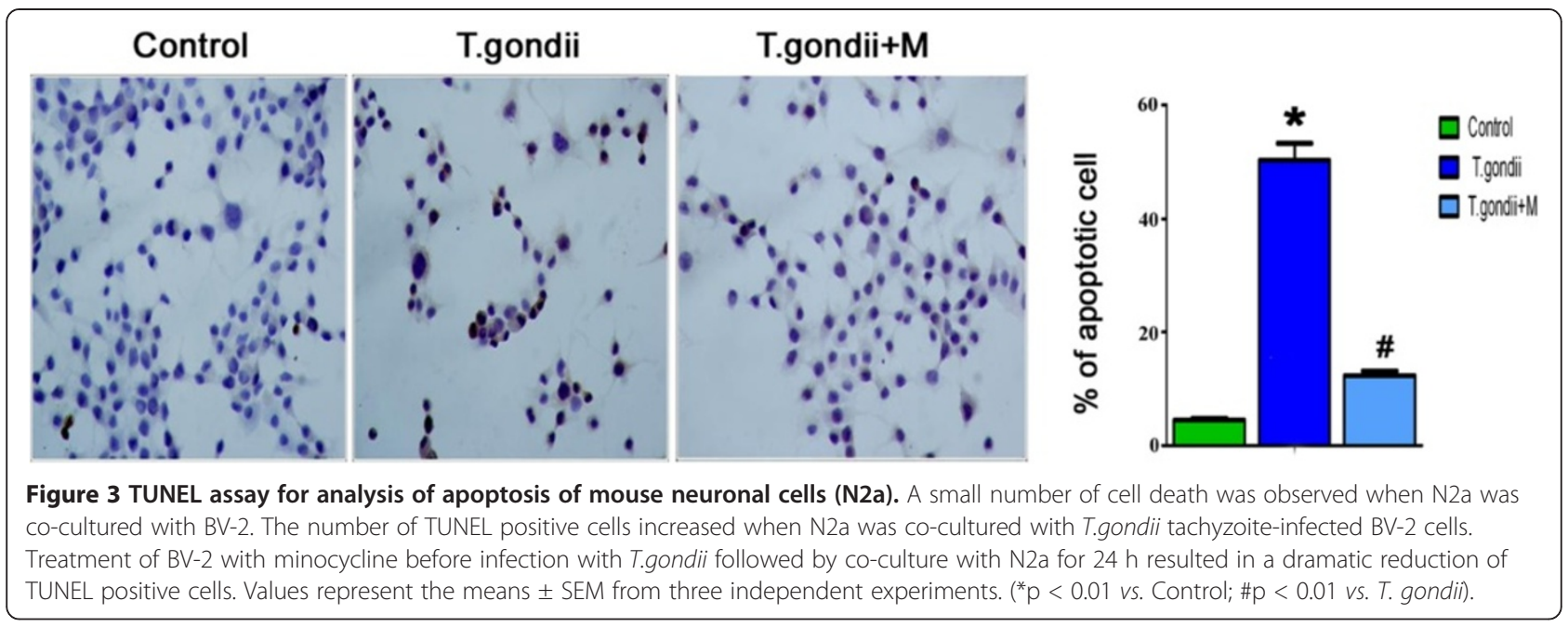

\section{Results}

\section{Toxoplasma encephalitis model was established}

Mice with latent Toxoplasma infection were immunologically compromised by cyclophosphamide administration for 15 days and, accordingly, TE pathology in the brain tissues of animals was observed. Histological and immunohistochemical analyses showed that necrosis (Figure 1A(2) and free tachyzoites (Figure 1A(4) were present in the cortex of immunosuppressed mice. Inflammatory infiltration of neutrophils and lymphocytes was seen in foci of free tachyzoites. Inversely, no infiltration of inflammatory cells in the surrounding tissues of

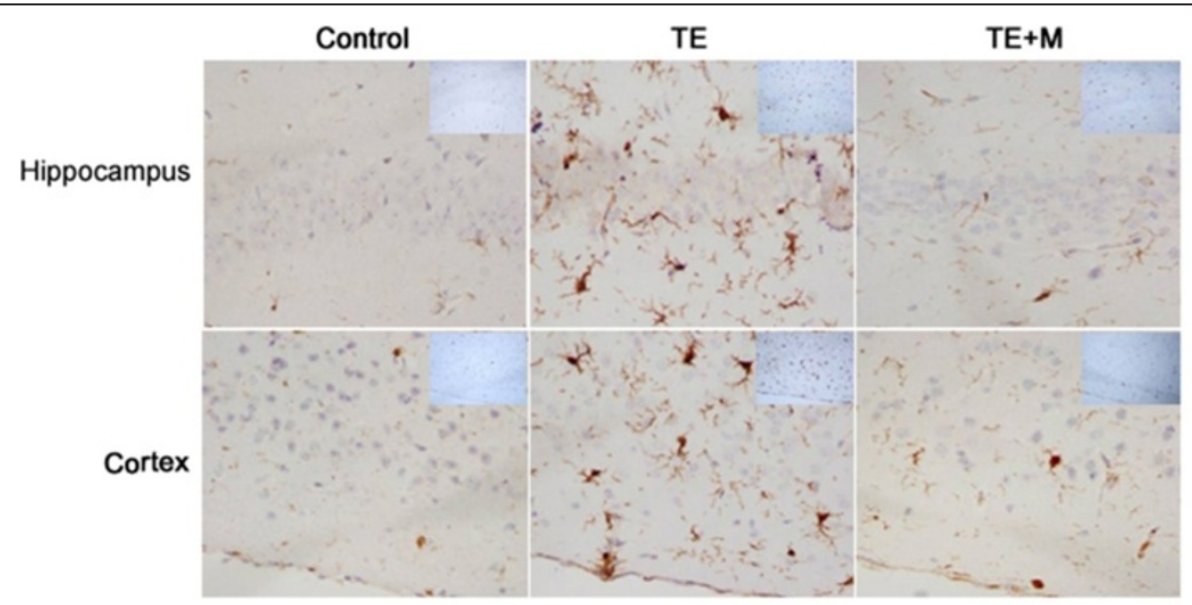

Hippocampus

Cortex
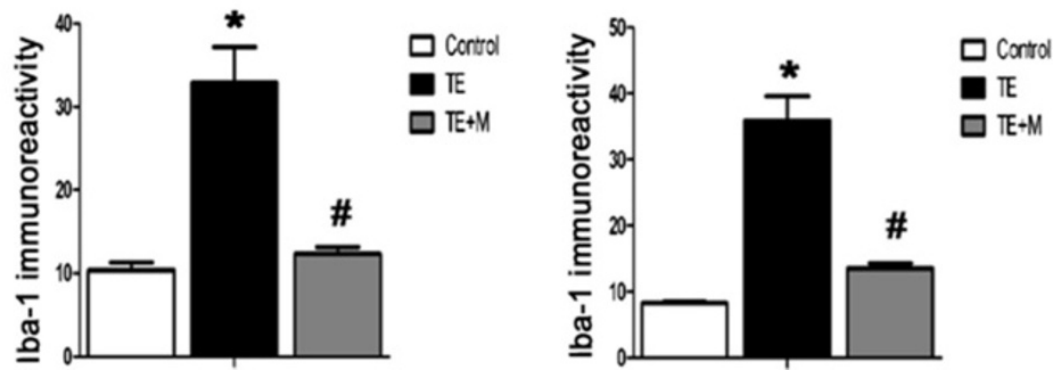

Figure 4 Immunocytochemical analysis of microglia in the brain of TE and TE $+\mathbf{M}$ mice. Brain sections from control, TE and TE $+\mathrm{M}$ animal groups were stained with polyclonal antibodies against Iba-1 (a specific microglial marker). Compared with control and TE + M mice, the number of Iba-1-positive cells in both hippocampal and cortex of TE mice was remarkably increased. Morphological characteristics of microglia activation were noted in TE. Minocycline treatment significantly inhibited microglial activation. Values represent the means \pm SEM of six animals per group. ( ${ }^{*} \mathrm{P}<0.01$ vs Control; \#P $<0.01$ vs TE). 
the single cyst was noted in the brain sections of mice with chronic Toxoplasma infection (Figure 1A(1) and A(3). Subsequently, we compared the parasite loads in the brain tissues of $\mathrm{TE}$ and $\mathrm{TE}+\mathrm{M}$ to exclude the parasiticide effect of minocycline. As a result, no statistical significance of Toxoplasma DNA quantification was seen between TE and TE $+M$ groups (data not shown). Hemiplegia, paraplegia, and other CNS injuries developed in mice with TE (Figure 1B).

\section{T. gondii infection induced neuronal apoptosis in TE that} was inhibited by minocycline treatment

To investigate the in vivo neuronal apoptosis in TE and the potential therapeutic effect of minocycline, fluorescence immunohistochemistry and Western blotting were performed to reveal the neuronal damage. The caspase-3 positive cells were observed in cortex of TE mice (Figure 2). The expression of NeuN (a specific neuron marker) of TE mice was significantly down-regulated when compared with the control (Figure 2). Accordingly, the in vitro studies also indicated that a low level of apoptosis of N2a cells was observed following co-culture or infection of N2a cells with parasites for $24 \mathrm{~h}$ (data not shown), suggesting that T.gondii inhibits rather than facilitates neuronal apoptosis. Apoptosis of N2a cells, however, dramatically increased when $\mathrm{N} 2 \mathrm{a}$ cells were co-cultured with BV-2 cells and the tachyzoites for $24 \mathrm{~h}$ (Figure 3 ). As expected, minocycline treatment significantly attenuated cellular apoptosis of $\mathrm{N} 2 \mathrm{a}$ in both in vivo and in vitro studies (Figures 2 and 3).

\section{Minocycline inhibited activation of microglia in cortex and hippocampus of mice with TE}

To investigate microglia activation in TE, antibodies against ionized calcium-binding adapter molecule-1 (Iba-1, a specific microglial marker) were used to label microglial cells. Immunohistochemistry analysis indicated that the number of Iba- 1 positive cells was significantly higher in TE than in $\mathrm{TE}+\mathrm{M}$ and control group (Figure 4). In the cortex and the hippocampus of TE mice, the microglia cells presented an
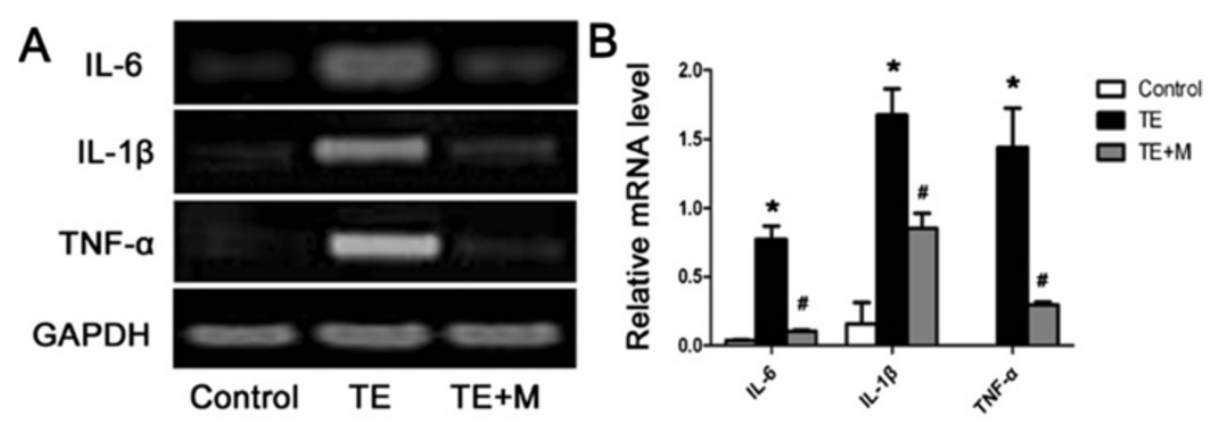

C
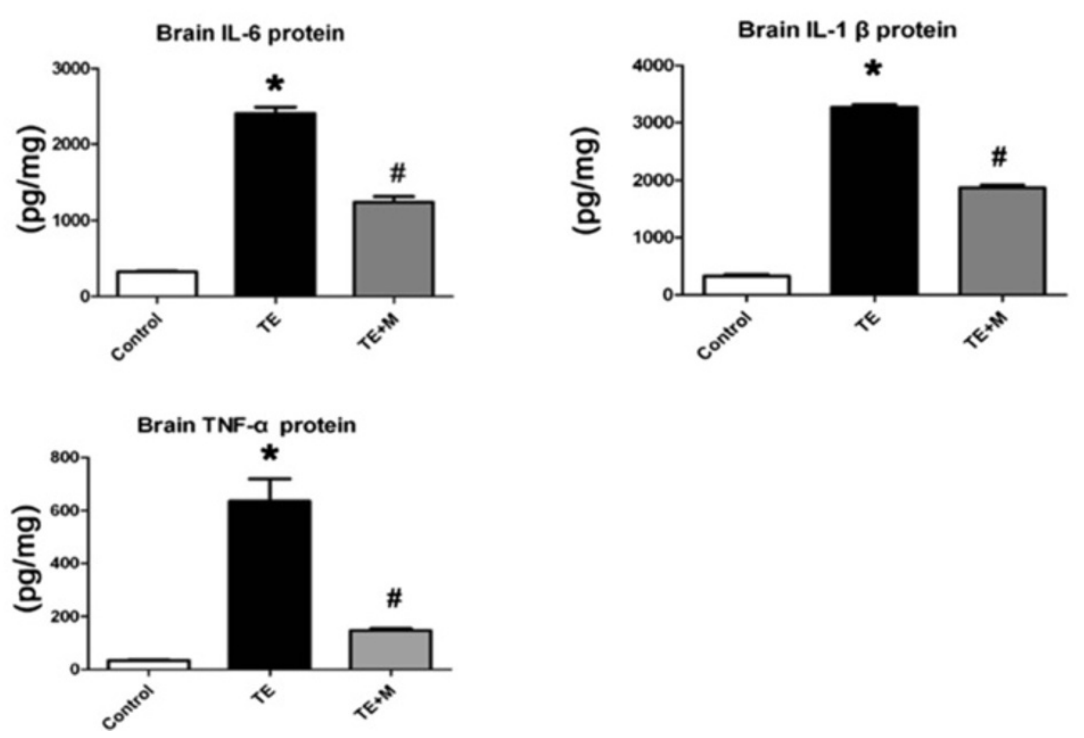

Figure 5 Expression of pro-inflammatory cytokines (IL-1 $\beta$, IL- 6 and TNF- $\alpha$ ) in the brain tissues of control, TE, and TE $+M$ mice. (A) Representative agarose-gel photographs show the expression level of $I L-1 \beta$, IL-6, and TNF-a mRNAs in brain tissues tested by quantitative RT-PCR. (B) Histograms represent densitometric quantitation of IL-1 $\beta$, IL-6, and TNF-a, normalized over GAPDH mRNA expression. (C) Expression of IL-1 $\beta$, IL-6, and TNF-a in brain tissues examined by ELISA. Values represent the means \pm SEM of six animals per group. ( ${ }^{*}, p<0.01$ vs. Control. \#, $p<0.01$ vs. TE). 
extensively branched process and hypertrophy of cell body, representing the morphological features of cell activation (Figure 4).

\section{T. gondii induced secretion of inflammatory cytokines that were inhibited by minocycline}

Activated microglia contribute to the production of inflammatory cytokines that mediate direct or indirect neuron death. Herein, we analyzed the inflammatory cytokines generated by activated microglia cells challenged with T.gondii tachyzoites. The qRT-PCR analysis indicated that expressions of interleukin-1 $\beta$ (IL-1 $\beta$ ), interleukin-6 (IL-6), and tumor necrosis factor- $\alpha$ (TNF- $\alpha$ ) mRNAs was remarkably enhanced in mice with TE, especially of IL-1 $\beta$ and TNF- $\alpha$ (Figure 5A and B), and the corresponding result was noted in the translational level when compared with the uninfected control (Figure 5C).

To verify the in vivo data that the inflammatory cytokines were induced by activated microglia in TE, mouse microglial cells of BV-2 were subjected to infection with Toxoplasma tachyzoites, and IL- $1 \beta$, IL-6, and TNF- $\alpha$ were detected with qRT-PCR and ELISA at $24 \mathrm{~h}$ postinfection. The results showed that expressions of IL-1 $\beta$, IL-6, and TNF- $\alpha$ were significantly elevated following parasite inoculation (Figure 6). Minocycline treatment notably restrained release of IL-1 $\beta$, IL-6, and TNF- $\alpha$

\section{A}
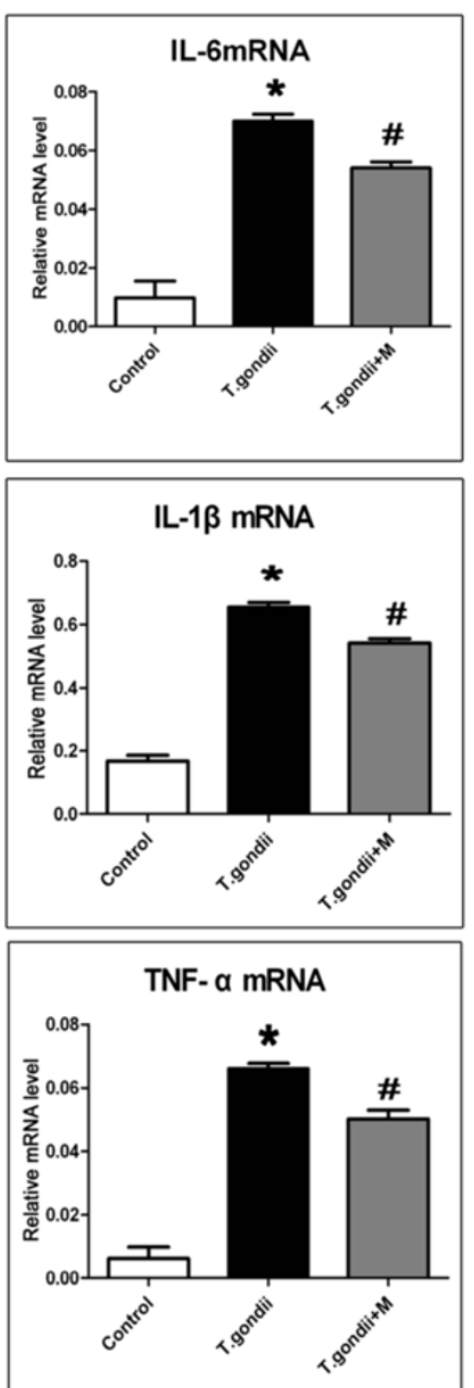

B
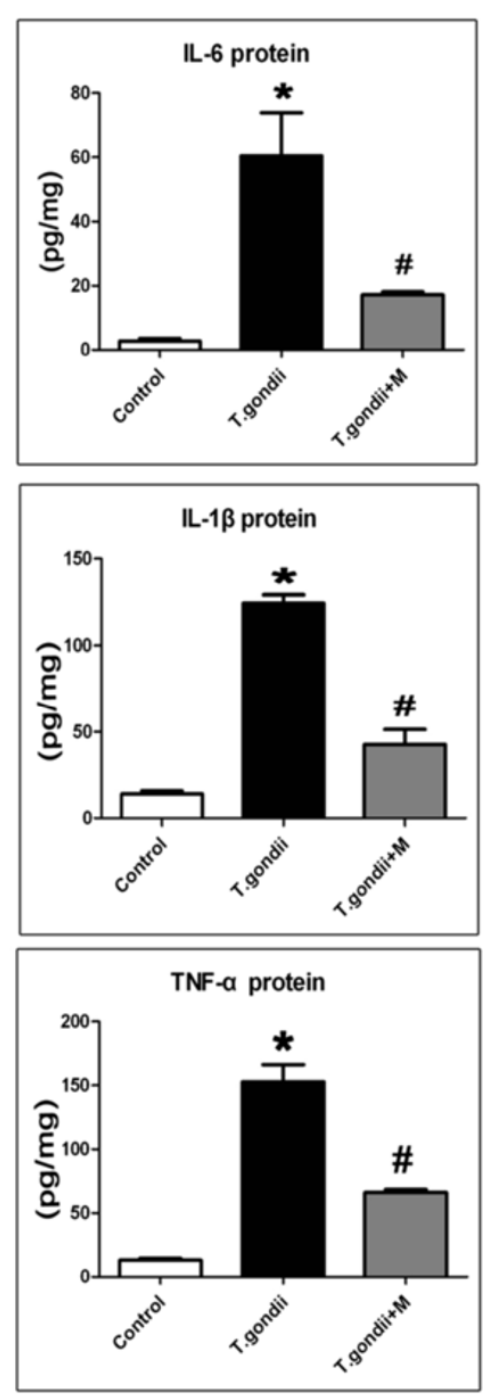

Figure 6 Pro-inflammatory cytokines (IL-1 $\beta$, IL-6, and TNF- $\boldsymbol{\alpha}$ ) produced by BV-2 cells. Mouse microglia BV-2 was either left uninfected, or T.gondii-infected (T.gondii) or T.gondii-infected and treated with minocycline (T.gondii + M) as described in the Methods section. (A) The mRNAs of pro-inflammatory cytokines were detected by quantitative RT-PCR. The relative levels of IL-1 $\beta, I L-6$, and TNF-a expression were compared with GAPDH. (B) Culture supernatants were collected after $24 \mathrm{~h}$ post-treatment and tested by ELISA to quantify IL-1 $\beta$, IL-6, and TNF-a secretion. The results represent the means \pm SEM of three independent experiments. ( ${ }^{*} P<0.01$ vs Control; \#P $<0.01$ vs T. gondii). 
secreted by activated microglia in both in vivo and in vitro experiments (Figures 5 and 6).

\section{T. gondii induced expression of iNOS in microglia which was blocked by minocycline}

Western blotting revealed a significant increase of expression of iNOS in TE mice compared with the uninfected group (Figure 7B). Double immunofluorescence staining demonstrated that iNOS was distinctly expressed by the Iba-1-immunoreactive cells rather than the neurons in the inflammatory foci (Figure 7A).

Immunofluorescence and Western blotting were used to detect the in vitro expression of iNOS in BV-2 cells challenged with tachyzoites(Figure 8). Similar to the in vivo approach, tachyzoites facilitated activation of microglia to express iNOS, while minocycline treatment significantly reduced iNOS production following T.gondii infection.

\section{Discussion}

Encephalitis caused by T.gondii is one of the lethal consequences in HIV/AIDS patients, and other immunocomprised individuals as well. So far knowledge has remained limited on the contributions of microglia to the pathogenesis of neuron damage in recrudescent toxoplasmosis. Results in the present study demonstrated that $T$. gondii induced microglia activation which upregulated pro-inflammatory cytokine expression and mediated neuronal death. Treatment with minocycline not only inhibited microglia activation and the expression of pro-inflammatory cytokines, but also attenuated the neuronal apoptosis. Our data suggest that microglia activation might play a crucial role in the pathogenesis of Toxoplasmic encephalitis (TE).

We hypothesized in the present study that neuron apoptosis noted in TE might not be, at least in part, a result from the primary injury by parasitism but from the

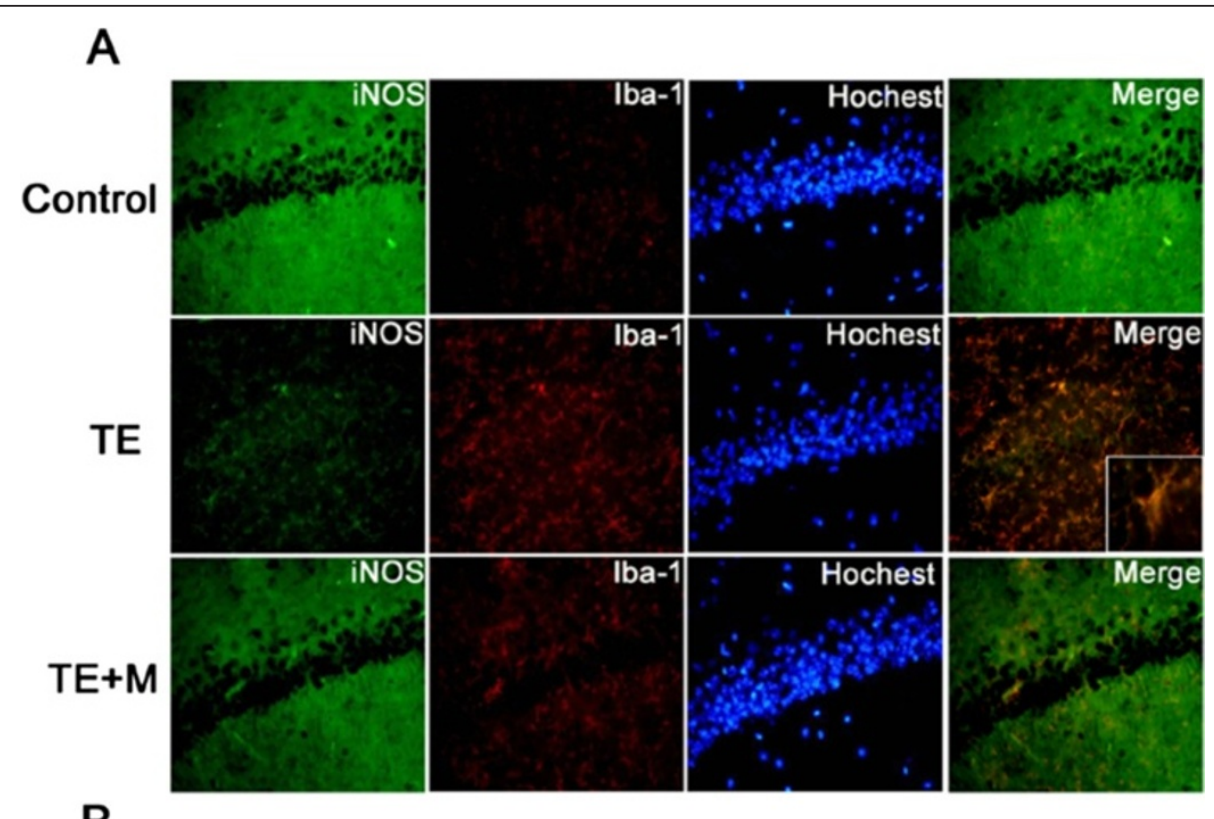

B
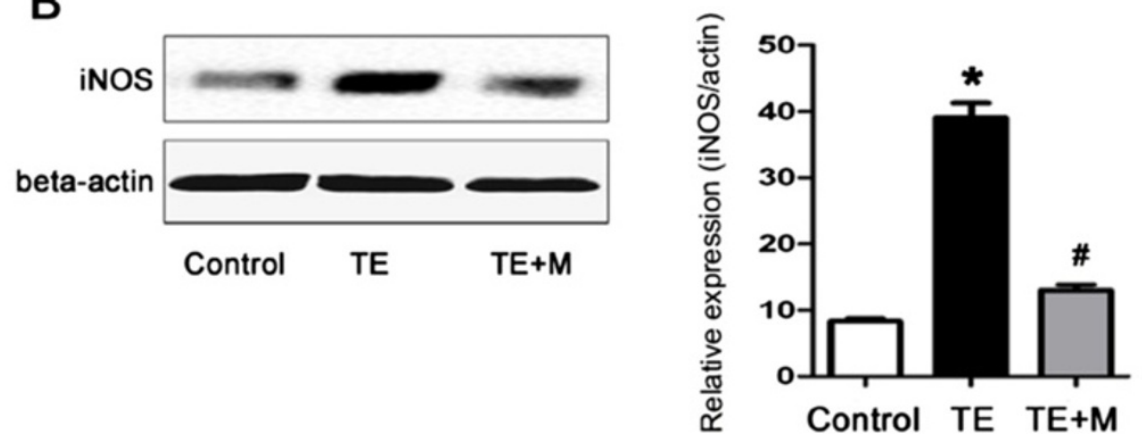

Figure 7 Expression of iNOS in brain of control, TE, and TE + M mice. (A) Double immunofluorescent staining of iNOS with Iba-1 (a specific marker of microglia). In the inflammatory loci of hippocampus, the number of iNOS-immunoreactive cells increased and the iNOS was expressed in microglia. (B) Western blotting analysis of iNOS expression. Histograms show densitometric analyses that revealed the relative levels of iNOS normalized over $\beta$-actin. Values represent the means \pm SEM of six animals per group. ( ${ }^{*} \mathrm{P}<0.01$ vs Control; \#P $<0.01$ vs TE). 


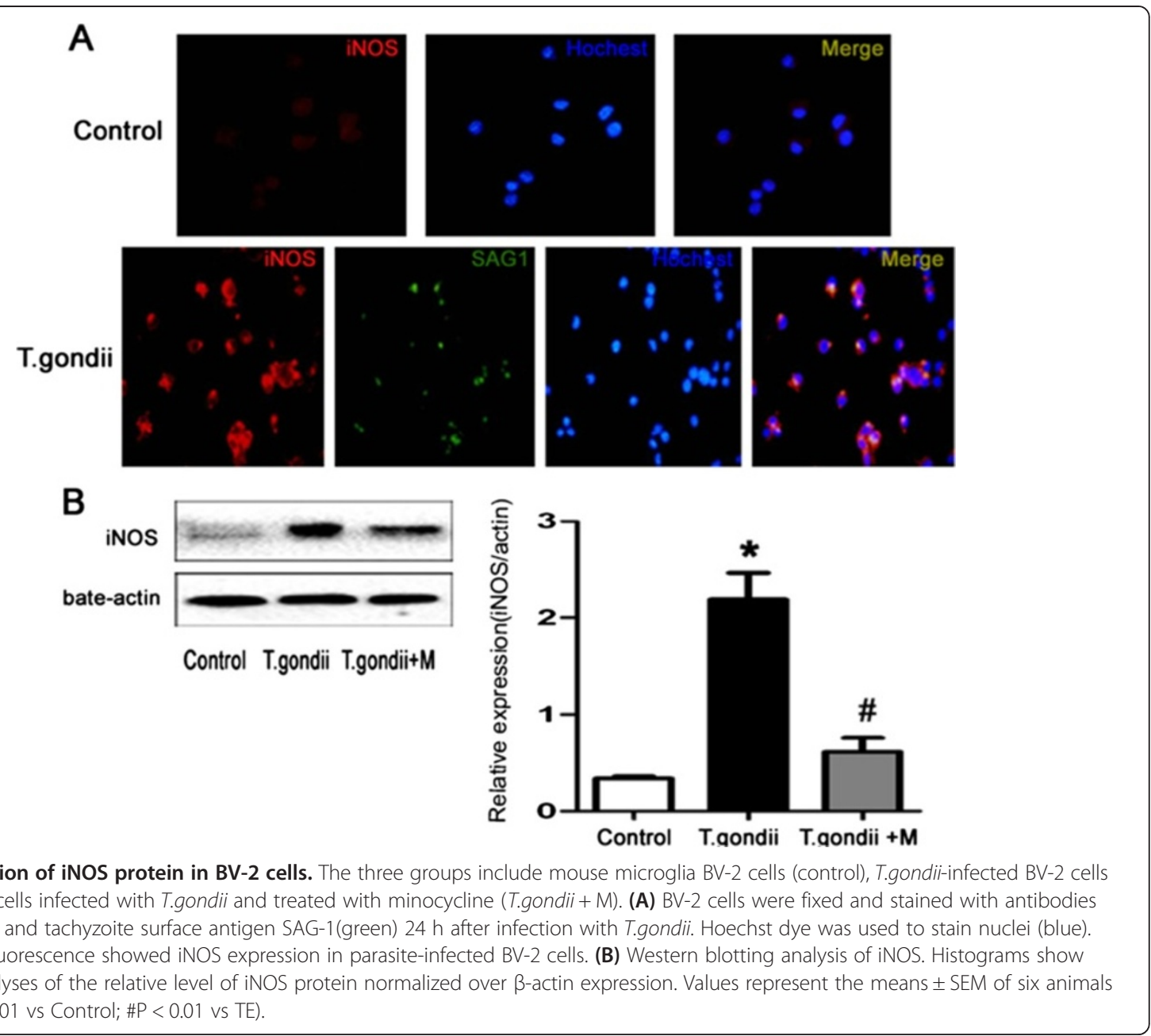

secondary response to microglia activation, which gives rise to the subsequent neuron cell damage, and is closely associated with the TE symptoms and signs [31].

Clinically, patients with TE exhibit severe neurological manifestations [32]. Previously, we found that reactivation of a latent Toxoplasma infection induced by cyclophosphamide led to hemiparesis and convulsions of mice, the pathological mechanism, however, remains unclarified. Although T.gondii readily invades neuron cells in vitro [33], interestingly, when $\mathrm{N} 2$ a cells were cocultured or infected with tachyzoites, only a small ratio of apoptotic cells were detectable. Previous investigations have shown that $T$. gondii could inhibit apoptosis of infected cells to ensure better dissemination within the host [4] and their own intracellular survival [34]. Several other studies, however, reported that $T$. gondiiinfected cells induced apoptosis of bystander cells by secretion of some soluble factors [21,35]. It is speculated that microglia may be involved in neuronal apoptosis in TE. As the resident macrophages of the CNS, microglia have extreme sensitivity to brain microenvironment stimuli, which responds to pathogen invasion or tissue damage by altering their morphology and phenotype. Similar to classically activated macrophages infected with Wh6 strain of type Chinese 1 [36], activated microglia can secrete a variety of pro-inflammatory and neurotoxic factors (e.g., IL-1 $\beta$, IL-6, and TNF- $\alpha$ ) and increase expression of iNOS. These factors are necessary to destroy invading pathogens and could directly induce apoptosis of host cells. Additionally, microglia activation is thought to be a causative factor in many neurological diseases including Alzheimer's disease, Parkinson's disease, and viral encephalitis [37]. These neural pathogeneses are generally associated with inflammatory mediators that are manifested in part by activated microglia. Relatively few studies have investigated the role of microglia in the pathogenesis of Toxoplasmic encephalitis. Herein, we observed that the activated microglia, as assessed by Iba- 1 immunoreactivity, is increased dramatically in the cortex and hippocampus with TE, which positively correlates with the dominant distribution of $T$. gondii cysts in the cortex and hippocampus of the mouse brain [38,39]. (Figures 1 and 4). 
Hippocampus and cortex are associated with cognitive and movement functions. Effects of Toxoplasma infection on human and animal behavior have been reported [40]. Here we noted the morphological alteration of microglia and increased expression of IL-1 $\beta$, IL- 6 , TNF- $\alpha$ in T.gondii infected microglial cells and, by using transwell culture, we also found that the apoptosis deteriorated when N2a cells were co-cultured in the presence of microglia and parasites (Figure 3). These findings may provide some explanations for the neural symptoms and signs of hosts with Toxoplasmic encephalitis, although the role of Toxoplasma effectors (e.g., dense granule protein 15, GRA15) in polarization of microglia remains to be elucidated in the isolates of genotype Chinese 1.

Minocycline is a semisynthetic tetracycline that has been shown to have neuroprotective effects in animal models of various neural disorders [41,42]. Minocycline is able to inhibit activation of macrophages and microglia, and thereby decrease inflammatory factor production (such as TNF- $\alpha$, IL-1 $\beta$, and IL-6) [43]. The present investigation indicates that minocycline significantly restrained microglia activation and, consequently, protected the neural cells from apoptosis in vivo. Additionally, minocycline remarkably reduced expression of IL-1 $\beta$, IL-6, TNF- $\alpha$, and iNOS. Apoptosis of N2a cells was significantly ameliorated when the co-cultured BV-2 cells were treated with minocycline. Furthermore, no statistically significant difference was seen between $\mathrm{TE}$ and $\mathrm{TE}+\mathrm{M}$ groups in parasite burden in the brain of mice, suggesting the inability of minocycline to directly affect the vitality of T.gondii.

\section{Conclusions}

Taken together, our findings reveal that T.gondii tachyzoites can strongly induce the secretion of inflammatory cytokines via activation of microglia both in vivo and in vitro. Inflammation generated by activated microglia is responsible for neuronal lesions in the brain of mice with reactivated Toxoplasmic encephalitis. The therapy of minocycline would help attenuate the pathology of mice with TE via inhibition of microglia activation and the causative cytokines involved.

\footnotetext{
Abbreviations

TE: Toxoplasmic encephalitis; CNS: Central nervous system; IL-6: Interleukin-6; IL-1ß: Interleukin-1 $\beta$; TNF-a: Tumor necrosis factor-a; iNOS: Inducible nitric oxide synthase; TBI: Traumatic brain injury; ICH: Intracerebral hemorrhage; LNB: Lyme neuroborreliosis; LPS: Lipopolysaccharide; GPIs:

Glycosylphosphatidylinositols; ROPs: Rhoptries; GRAs: Dense granules.
}

\section{Competing interests}

All authors declare that they have no competing interests in the work.

\section{Authors' contributions}

Conceived and designed the study: JLS, ZRL, XLW, YHZ. Performed the experiments: YHZ, HC, RA, YC, LW. Analyzed the data: YHZ, ML. Animal handling: QLL, YHC. Wrote the paper: YHZ, HC, JD. Paper revision: YHX, JLS. All authors read and approved the final manuscript.
Authors' information

Yi-hua Zhang and He Chen are co-first authors.

\section{Acknowledgements}

This work was funded by the National Basic Science Research Program of China (Grant No. 2010CB530001), the National Natural Science Foundation of China (Grant No.81471983; 81101272; 81271864), Fok Ying Tung College Young Teachers Fund (131033) of the Chinese Ministry of Education and the Outstanding Youth Fund of Anhui Province (10040606Y19). We thank Dr. Wen-qi Liu at Tongji Medical School, Huazhong Univisity of Science and Technology, for her assistance in parasite isolation. Our gratitude is given to Dr. SH Huang at The School of Life Sciences, Anhui Medical University, for his kind gift of BV-2and N2a cells.

\section{Author details}

${ }^{1}$ The Key Laboratory of Zoonoses and Pathogen Biology Anhui, and Department of Parasitology, Anhui Medical University, Hefei, China. ${ }^{2}$ Clinical Laboratory, the First Affiliated Hospital of Anhui Medical University, Hefei, Anhui, China. ${ }^{3}$ Department of Blood Transfusion, the First Affiliated Hospital of Anhui Medical University, Hefei, Anhui, China. ${ }^{4}$ Department of Biochemistry and Molecular Biology, School of Basic Medicine, Anhui Medical University, Hefei, China. ${ }^{5}$ State Key Laboratory of Biocontrol and Center for Parasitic Organisms, School of Life Sciences, Zhongshan School of Medicine, Sun Yat-Sen University, Guangzhou, China.

Received: 13 March 2014 Accepted: 1 August 2014

Published: 15 August 2014

\section{References}

1. Tenter AM, Heckeroth AR, Weiss LM: Toxoplasma gondii: from animals to humans. Int J Parasitol 2000, 30:1217-1258.

2. Weiss LM, Dubey JP: Toxoplasmosis: a history of clinical observations. Int J Parasitol 2009, 39:895-901.

3. Jones JL, Hanson DL, Chu SY, Ciesielski CA, Kaplan JE, Ward JW, Thomas R: Navin and the Adult/Adolescent Spectrum of Disease Group: Toxoplasmic encephalitis in HIV-infected persons: risk factors and trends. AIDS 1996, 10:1393-1399.

4. Dellacasa-Lindberg I, Fuks JM, Arrighi RBG, Lambert H, Wallin RPA, Chambers BJ, Barragan A: Migratory Activation of Primary Cortical Microglia upon Infection with Toxoplasma gondii. Infect Immun 2011, 79:3046-3052

5. Reiter-Owona I, Seitz H, Gross U, Sahm M, Rockstroh JK, Seitz HM: Is stage conversion the initiating event for reactivation of Toxoplasma gondii in brain tissue of AIDS patients? J Parasitol 2000, 86:531-536.

6. Hill D, Dubey JP: Toxoplasma gondii: transmission, diagnosis and prevention. Clin Microbiol Infect 2002, 8:634-640.

7. Hermes G, Ajioka JW, Kelly KA, Mui E, Roberts F, Kasza K, Mayr T, Kirisits MJ, Wollmann R, Ferguson DJ, Roberts CW, Hwang JH, Trendler T, Kennan RP, Suzuki Y, Reardon C, Hickey WF, Chen L, McLeod R: Neurological and behavioral abnormalities, ventricular dilatation, altered cellular functions, inflammation, and neuronal injury in brains of mice due to common, persistent, parasitic infection. J Neuroinflammation 2008, 5:48.

8. Fischer H-G, Reichmann G: Brain dendritic cells and macrophages/microglia in central nervous system inflammation. J Immunol 2001, 166:2717-2726.

9. Aloisi F: Immune function of microglia. Glia 2001, 36:165-179.

10. Glass CK, Saijo K, Winner B, Marchetto MC, Gage FH: Mechanisms underlying inflammation in neurodegeneration. Cell 2010, 140:918-934.

11. Liu B, Gao HM, Wang JY, Jeohn GH, Cooper CL, Hong JS: Role of nitric oxide in inflammation-mediated neurodegeneration. Ann N Y Acad Sci 2002, 962:318-331.

12. Jensen KD, Wang Y, Wojno ED, Shastri AJ, Hu K, Cornel L, Boedec E, Ong YC, Chien YH, Hunter CA, Boothroyd JC, Saeij JP: Toxoplasma polymorphic effectors determine macrophage polarization and intestinal inflammation. Cell Host Microbe 2011, 9:472-483.

13. Loane DJ, Byrnes KR: Role of microglia in neurotrauma. Neurotherapeutics 2010, 7:366-377.

14. Dohi K, Ohtaki H, Nakamachi T, Yofu S, Satoh K, Miyamoto K, Song D, Tsunawaki S, Shioda S, Aruga T: Gp91 phox (NOX2) in classically activated microglia exacerbates traumatic brain injury. J Neuroinflammation 2010, $7: 41$. 
15. Lin S, Yin Q, Zhong Q, Lv FL, Zhou Y, Li JQ, Wang JZ, Su BY, Yang QW: Heme activates TLR4-mediated inflammatory injury via MyD88/TRIF signaling pathway in intracerebral hemorrhage. J Neuroinflammation 2012, 9:46

16. Liu M, Cai T, Zhao F, Zheng G, Wang Q, Chen Y, Huang C, Luo W, Chen J: Effect of microglia activation on dopaminergic neuronal injury induced by manganese, and its possible mechanism. Neurotox Res 2009, 16:42-49.

17. Ramesh G, Borda JT, Dufour J, Kaushal D, Ramamoorthy R, Lackner AA, Philipp MT: Interaction of the Lyme disease Spirochete Borrelia burgdorferi with brain parenchyma elicits inflammatory mediators from glial cells as well as glial and neuronal apoptosis. Am J Pathol 2008, 173:1415-1427.

18. Debierre-Grockiego F, Campos MA, Azzouz N, Schmidt J, Bieker U, Resende MG, Mansur DS, Weingart R, Schmidt RR, Golenbock DT, Gazzinelli RT, Schwarz RT: Activation of TLR2 and TLR4 by glycosylphosphatidylinositols derived from Toxoplasma gondii. J Immunol 2007, 179:1129-1137.

19. Murray PJ: Macrophages as a battleground for toxoplasma pathogenesis. Cell Host Micro-be 2011, 9:445-447.

20. Kim L, Denkers EY: Toxoplasma gondii triggers Gi-dependent PI3-kinase signaling required for inhibition of host cell apoptosis. J Cell Sci 2006, 119:2119-2126.

21. Nishikawa $Y$, Kawase $O$, Vielemeyer $O$, Suzuki $H$, Joiner $K A$, Xuan $X$ Nagasawa $\mathrm{H}$ : Toxoplasma gondii infection induces apoptosis in noninfected macrophages: role of nitric oxide and othersoluble factors. Parasite Immunol 2007, 29:375-385.

22. Hunter CA, Abrams JS, Beaman MH, Remington JS: Cytokine mRNA in the central nervous system of SCID mice infected with Toxoplasma gondii: importance of T-cell-independent regulation of resistance to T. gondii. Infect Immun 1993, 61:4038-4044.

23. Nebuloni M, Pellegrinelli A, Ferri A, Tosoni A, Bonetto S, Zerbi P, Boldorini R, Vago L, Costanzi G: Etiology of microglial nodules in brains of patients with acquired immunodeficiency syndrome. J Neurovirol 2000, 6:46-50.

24. Chen ZW, Gao JM, Huo XX, Wang L, Yu L, Halm-Lai F, Xu YH, Song WJ, Hide $G$, Shen JL, Lun ZR: Genotyping of Toxoplasma gondii isolates from cats in different geographic regions of China. Vet Parasitol 2011, 183:166-170.

25. Wang $Y$, Meng Q, Qiao $H$, Jiang $H$, Sun X: Role of the spleen in cyclophosphamide-induced hematosuppression and extramedullary hemato- poiesis in mice. Arch Med Res 2009, 40:249-255

26. Sumyuen MH, Garin YJ, Derouin F: Effect of immunosuppressive drug regimens on acute and chronic murine toxoplasmosis. Parasitol Res 1996, 82:681-686

27. Chen L, Shi W, Li H, Sun X, Fan X, Lesage G, Li H, Li Y, Zhang Y, Zhang X, Zhang $Y$, Yin D: Critical role of toll-like receptor 9 in morphine and My- cobacterium tuberculosis-induced apoptosis in mice. PLoS One 2010, 5:e9205.

28. Kaplan JE, Benson C, Holmes KH, Brooks JT, Pau A, Masur H: Guidelines for prevention and treatment of opportunistic infections in HIV- infected adults and adolescents: recommendations from CDC, the National Institutes of Health, and the HIV Medicine Association of the Infectious Diseases Society of America. MMWR Recomm Rep 2009, 58:1-207.

29. Liang K, Jiang Z, Ding BQ, Cheng P, Huang DK, Tao LM: Expression of cell proliferation and apoptosis biomarkers in pterygia and normal conjunctiva. Mol Vis 2011, 17:1687-1693.

30. Zhang GH, Lv MM, Wang S, Chen L, Qian NS, Tang Y, Zhang XD, Ren PC, Gao CJ, Sun XD, Xu LX: Spinal astrocytic activation is involved in a virally- induced rat model of neuropathic pain. PLoS One 2011, 6:e23059.

31. Händel U1, Brunn A, Drögemüller K, Müller W, Deckert M, Schlüter D: Neuronal gp130 expression is crucial to prevent neuronal loss, hyperinflammation, and lethal course of murineToxoplasma encephalitis. Am J Pathol 2012, 181(1):163-173.

32. Davis C, Manteuffel J: Unusual presentation of Toxoplasma gondii encephalitis. West J Emerg Med 2012, 13:488-490.

33. Fischer $H G$, Nitzgen B, Reichmann G, Gross U, Hadding U: Host cells of Toxoplasma gondii encystation in infected primary culture from mouse brain. Parasitol Res 1997, 83:637-641.

34. Cai $Y$, Chen $H$, Jin L, You Y, Shen JL: STAT3-dependent transactivation of miRNA genes following Toxoplasma gondii infection in macrophage. Parasit Vectors 2013, 6:356.

35. Xu X, Liu T, Zhang A, Huo X, Luo Q, Chen Z, Yu L, Li Q, Liu L, Lun ZR, Shen $\mathrm{J}$ : Reactive oxygen species-triggered trophoblast apoptosis is initiated by endoplasmic reticulum stress via activation of caspase-12, $\mathrm{CHOP}$, and the JNK pathway in Toxoplasma gondii infection in mice. Infect Immun 2012, 80:2121-2132

36. Zhang AM, Shen Q, Li M, Xu XC, Chen H, Cai YH, Luo QL, Chu DY, Yu L, Du J, Lun ZR, Wang Y, Sha Q, Shen JL: Comparative studies of acrophagebiased responses in mice to infection with Toxoplasma gondii ToxoDB \#9 strains of different virulence isolated from China. Parasit Vectors 2013, 6:308.

37. Ghoshal A, Das S, Ghosh S, Mishra MK, Sharma V, Koli P, Sen E, Basu A: Proinflammatory mediators released by activated microglia induces neuronal death in Japanese encephalitis. Glia 2007, 55:483-496.

38. Dellacasa-Lindberg I, Hitziger N, Barragan A: Localized recrudescence of Toxoplasma infections in the central nervous system of immunocompromised mice assessed by in vivo bioluminescence imaging. Microbes Infect 2007, 9:1291-1298.

39. Berenreiterova M, Flegr J, Kube NAAA, Nemec P: The distribution of Toxoplasma gondii cysts in the brain of a mouse with latent Toxoplasmosis: implications for the behavioral manipulation hypothesis. PLoS One 2011, 6(12):e28925.

40. Caselli D, Andreoli E, Paolicchi O, Savelli S, Guidi S, Pecile P, Aricò M: Acute encephalopathy in the immune-compromised child: never forget toxo- plasmosis. J Pediatr Hematol Oncol 2012, 34:383-386.

41. Tang M, Alexander $H$, Clark RS, Kochanek PM, Kagan VE, Bayir H: Minocycline reduces neuronal death and attenuates microglial response after pediatric asphyxial cardiac arrest. J Cereb Blood Flow Metab 2010, 30:119-129.

42. Abraham J, Fox PD, Condello C, Bartolini A, Koh S: Minocycline attenuates microglia activation and blocks the long-term epileptogenic effects of early-life seizures. Neurobiol Dis 2012, 46:425-430.

43. Henry CJ, Huang Y, Wynne A, Hanke M, Himler J, Bailey MT, Sheridan JF, Godbout JP: Minocycline attenuates lipopolysaccharide (LPS)-induced neuroinflammation, sickness behavior, and anhedonia. J Neuroinflammation 2008, 5:15.

doi:10.1186/1756-3305-7-372

Cite this article as: Zhang et al:: Activated microglia contribute to neuronal apoptosis in Toxoplasmic encephalitis. Parasites \& Vectors 2014 7:372

\section{Submit your next manuscript to BioMed Central and take full advantage of:}

- Convenient online submission

- Thorough peer review

- No space constraints or color figure charges

- Immediate publication on acceptance

- Inclusion in PubMed, CAS, Scopus and Google Scholar

- Research which is freely available for redistribution 